\title{
E3 Ubiquitin-Protein Ligase CHFR
}

National Cancer Institute

\section{Source}

National Cancer Institute. E3 Ubiquitin-Protein Ligase CHFR. NCI Thesaurus. Code

C77211.

E3 ubiquitin-protein ligase CHFR (664 aa, 73 kDa) is encoded by the human CHFR gene.

This protein is involved in both ubiquitination of proteins and the mitotic checkpoint. 